\title{
OSCILLATION THEOREMS FOR NONLINEAR SECOND ORDER DIFFERENCE EQUATIONS WITH A NONLINEAR DAMPING TERM
}

\author{
E. THANDAPANI AND S. PANDIAN
}

\begin{abstract}
Albstract. Some new sufficient conditions for the oscillation of the nonlinear damped second order difference equations of the form

$$
\Delta^{2} y_{n}+p_{n} \phi\left(y_{n}, \Delta y_{n}\right)+q_{n} f\left(y_{n+1}\right) g\left(\Delta y_{n}\right)=0
$$

are given.
\end{abstract}

\section{Introduction}

In this paper we are concerned with the second order non-linear damped difference equation

$$
\Delta^{2} y_{n}+p_{n} \phi\left(y_{n}, \Delta y_{n}\right)+q_{n} f\left(y_{n+1}\right) g\left(\Delta y_{n}\right)=0, \quad n \in Z
$$

where $Z=\{1,2, \cdots\},\left\{p_{n}\right\}$ and $\left\{q_{n}\right\}$ are real sequences, the functions $f$ and $g$ are defined on the set $R$ of real numbers, the function $\phi$ is defined on $R \times R$ and $\Delta$ is the forward difference operator, defined by $\Delta y_{n}=y_{n+1}-y_{n}$. It is interesting to study second order nonlinear difference equations because they have physical applications as evidenced by [1].

Throughout the paper the term solution of (1) is always used for a sequence $\left\{y_{n}\right\}$ which satisfies (1) for all $n \in Z$ and for which $\sup \left\{\left|y_{n}\right|: n \geq s\right\}>0$ for any $s \in Z$. We make the standing hypothesis that equation (1) does possess such solutions. A solution is called non-oscillatory if it is eventually of constant sign. Otherwise it is called oscillatory.

Recently there has been an increasing interest in the study of the qualitative behaviour of solutions of difference equations of type (1) and /or related equations, see for example $[1,4,5,7-10]$ and the references cited therein.

Received October 14, 1993, revised June 9, 1994.

1991 Mathematics Subject Classification. 39A12

Key words and phrases. Difference equation, Nonlinear damped term, Oscillation. 
The purpose of this paper is to present theorems that give sufficient conditions for all solutions of (1) to be oscillatory. The obtained results are discrete analogues of some known theorems for non-linear differential equations due to Baker [2], Grace and Lalli [3] and Naito [6].

Now, for the difference equation (1) each result we shall prove, requires some of the following conditions.

(c) $p_{n} \geq 0$ and $q_{n}>0$ for all $n \in Z$;

$\left(c_{2}\right)$ there exists a constant $M>0$ such that

$$
0<\phi(x, y) y \leq M y^{2} \text { for all } y \in R /\{0\} \text { and } x \in R
$$

(c) $y f(y)>0$ and $g(y)>0$ for all $y \neq 0$;

$\left(c_{4}\right) f$ is non-decreasing and

$$
f(-x y) \geq f(x y) \geq K_{1} f(x) f(y) \text { for } x, y>0 \text { for some constant } K_{1}>0
$$

$\left(c_{5}\right) g$ is non-increasing and

$$
g(-x y) \geq g(x y) \geq K_{2} g(x) g(y) \text { for } x, y>0 \text { for some constant } K_{2}>0
$$

(c6) $f(y) g(y) / y^{\alpha} \geq \beta>0$ for $y \neq 0$ where $\alpha$ is the ratio of odd positive integers.

In the sequel we need the following two Lemmas of which the first Lemma is due to Hooker and Patula [4] and the second Lemma is a discrete analogue of Baker [2].

Lemma 1. If $y_{N} \geq 0, \Delta^{2} y_{n} \leq 0$ and $\Delta y_{n}>0$ for $n \geq N$, then $y_{n+1} \geq$ $\frac{n}{2} \Delta y_{n}$ for all $n \geq 2 N$.

Lemma 2. Suppose $\left(c_{1}\right)-\left(c_{3}\right)$ hold. Further assume

$$
1-M p_{n}>0 \text { for all } n \in N
$$

and

$$
\sum_{n=N}^{\infty}\left(\prod_{s=N}^{n-1}\left(1-M p_{s}\right)\right)=\infty
$$

Then, if $\left\{y_{n}\right\}$ is a non-oscillatory solution of (1), we have $y_{n} \Delta y_{n}>0$ for all sufficiently large $n \geq N$.

Proof. Let $\left\{y_{n}\right\}$ be a non-oscillatory solution of equation (1) and assume $y_{n}>0$ for $n \geq n_{0} \in Z$. Suppose $\left\{\Delta y_{n}\right\}$ is oscillatory, then there exists an integer $n_{1} \geq n_{0} \geq 0$ such that $\Delta y_{n_{1}}<0$ or $\Delta y_{n_{1}}=0$. First we consider $\Delta y_{n_{1}}<0$. Now, from equation $(1)$

$$
\Delta y_{n_{1}} \Delta^{2} y_{n_{1}}+p_{n_{1}} \phi\left(y_{n_{1}}, \Delta y_{n_{1}}\right) \Delta y_{n_{1}}=-q_{n_{1}} f\left(y_{n_{1}+1}\right) g\left(\Delta y_{n_{1}}\right) \Delta y_{n_{1}}>0
$$


or

$$
\begin{aligned}
& \Delta y_{n_{1}} \Delta^{2} y_{n_{1}}>-p_{n_{1}} \phi\left(y_{n_{1}}, \Delta y_{n_{1}}\right) \Delta y_{n_{1}} \\
& \Delta y_{n_{1}}\left(\Delta y_{n_{1}+1}-\Delta y_{n_{1}}\right)>-p_{n_{1}} M\left(\Delta y_{n_{1}}\right)^{2}
\end{aligned}
$$

and hence

$$
\Delta y_{n_{1}} \Delta y_{n_{1}+1}>\left(1-M p_{n_{1}}\right)\left(\Delta y_{n_{1}}\right)^{2}>0
$$

Dividing the above inequality by the negative term $\Delta y_{n_{1}}$, we obtain

$$
\Delta y_{n_{1}+1}<0
$$

By induction, we obtain

$$
\Delta y_{n}<0 \text { for all } n \geq n_{1} \in Z \text {. }
$$

Suppose $\Delta y_{n_{1}}=0$, then equation (1) implies

$$
\Delta y_{n_{1}+1}<0
$$

and we obtain as above

$$
\Delta y_{n}<0 \text { for all } n>n_{1} \in Z \text {. }
$$

Hence, in both the cases, we obtain

$$
\Delta y_{n}<0 \text { for all } n \geq n_{1}
$$

which however contradicts the assumption that $\left\{\Delta y_{n}\right\}$ oscillates. Thus $\left\{\Delta y_{n}\right\}$ is of fixed sign.

Let $\Delta y_{n}<0$ for all $n \geq N \in Z$.

From (1) and $\left(c_{2}\right)$, we obtain,

$$
\Delta u_{n}+M p_{n} u_{n} \geq 0
$$

where $u_{n}=-\Delta y_{n}$. From (4) we have

$$
u_{n} \geq u_{N} \prod_{s=N}^{n-1}\left(1-M p_{s}\right)
$$

or

$$
\Delta y_{n} \leq-u_{N} \prod_{s=N}^{n-1}\left(1-M p_{s}\right) .
$$

Summing the last inequality from $N$ to $(n-1)$, we obtain

$$
y_{n} \leq y_{N}-u_{N} \sum_{s=N}^{n-1} \prod_{t=N}^{s-1}\left(1-M p_{t}\right)
$$


In view of (3), we get

$$
y_{n} \rightarrow-\infty \text { as } n \rightarrow \infty \text {, a contradiction }
$$

Remark. Note that when $p_{n}=0$, conditions (2) and (3) are automatically satisfied.

\section{Main Results}

In this section we establish sufficient conditions for the oscillation of all solutions of equation (1). We begin with the following theorem.

Theorem 1. In addition to conditions $\left(c_{1}\right)-\left(c_{4}\right),(2)$ and $(3)$, suppose $f(u) g(u)$ is non-decreasing for $u \neq 0$,

$$
\int_{+0} \frac{d u}{f(u) g(u)}<\infty \text { and } \int_{-0} \frac{d u}{f(u) g(u)}<\infty
$$

and

$$
\sum^{\infty} q_{n} f(n)=\infty
$$

Then every solution of equation (1) is oscillatory.

Proof. Suppose that $\left\{y_{n}\right\}$ is a non-oscillatory solution of (1). In view of Lemma 2 , there is no loss in generality in assuming that there is an integer $N$ such that

$y_{n}>0$ and $\Delta y_{n}>0$ for all $n \geq N$. Since this implies that $\phi\left(y_{n}, \Delta y_{n}\right)>0$ for all $n \geq N$, it follows that

$$
\Delta^{2} y_{n}+q_{n} f\left(y_{n+1}\right) g\left(\Delta y_{n}\right) \leq 0 .
$$

Since $f$ is non-decreasing, we have from Lemma 1

$$
f\left(y_{n+1}\right) \geq f\left(\frac{n}{2} \Delta y_{n}\right) \text { for all } n \geq 2 N .
$$

Using (9) in (8) and then applying condition $\left(c_{4}\right)$ we obtain

$$
\Delta^{2} y_{n}+K_{1}^{2} f\left(\frac{1}{2}\right) q_{n} f(n) f\left(\Delta y_{n}\right) g\left(\Delta y_{n}\right) \leq 0
$$

or

$$
\frac{\Delta^{2} y_{n}}{f\left(\Delta y_{n}\right) g\left(\Delta y_{n}\right)}+K_{1}^{2} f\left(\frac{1}{2}\right) q_{n} f(n) \leq 0 \text { for all } n \geq 2 N \text {. }
$$

Observe $\Delta y_{n} \geq x \geq \Delta y_{n+1}$, we have

$$
f(x) g(x) \leq f\left(\Delta y_{n}\right) g\left(\Delta y_{n}\right)
$$


and therefore

$$
\frac{\Delta^{2} y_{n}}{f(x) g(x)} \leq \frac{\Delta^{2} y_{n}}{f\left(\Delta y_{n}\right) g\left(\Delta y_{n}\right)}
$$

Using the above inequality in (10) and summing from $2 N$ to $n$, we obtain

$$
\begin{aligned}
K_{1}^{2} f\left(\frac{1}{2}\right) \sum_{s=2 N}^{n} q_{s} f(s) & \leq \sum_{s=2 N}^{n-1} \int_{\Delta y_{s+1}}^{\Delta y_{s}} \frac{d x}{f(x) g(x)} \\
& \leq \int_{y_{n}}^{y_{2 N}} \frac{d x}{f(x) g(x)}<\infty
\end{aligned}
$$

which contradicts (7) and this proves the theorem.

Remark. In Theorem 1,

a) if $p_{n}=0$, then it reduces to Theorem 3 of [10],

b) if $p_{n}=0$ and $g(u) \equiv 1$, then it reduces to a special case of Theorem 4.1 of Kulenovic and Budincevic [5],

c) if $p_{n}=0, g(u) \equiv 1$ and $f(u)=u^{\alpha}, 0<\alpha<1$ then it reduces to Theorem 4.3 of Hooker and Patula [4].

Consider the difference equation

$$
\Delta^{2} y_{n}+\frac{1}{n} \Delta y_{n}+\frac{3(2)^{2 n-1 / 3}(3 n-1)}{n\left(1+3^{2 / 3}(2)^{2 n / 3}\right)} f\left(y_{n+1}\right) g\left(\Delta y_{n}\right)=0 \quad(n \in Z)
$$

where $f(u)=u^{1 / 3}$ and $g(u)=1+u^{2 / 3}$, then all conditions of Theorem 1 are satisfied. Hence, every solution of $\left(E_{1}\right)$ is oscillatory; one such solution is $y_{n}=(-1)^{n} 2^{n}$.

Corollary 2. Let the conditions (5) and (6) in Theorem 1 be replaced by the condition $\left(c_{6}\right)$ with $\alpha \in(0,1)$. Then every solution of equation (1) is oscillatory.

Proof. The proof is similar to that of Theorem 1 and hence is omitted.

Theorem 3. In addition to conditions $\left(c_{1}\right)-\left(c_{3}\right),\left(c_{5}\right),(2),(3)$ and (5), suppose that

$$
\int_{c}^{ \pm \infty} \frac{d x}{f(x) g(x)}<\infty \text { for all } c>0
$$

and

$$
\sum^{\infty} n q_{n} g\left(\frac{2}{n}\right)=\infty
$$

Then all solutions of equation (1) are oscillatory.

Proof. Suppose that $\left\{y_{n}\right\}$ is a non-oscillatory solution of (1). As before, we may assume that there is an integer $N \geq 0$ such that

$$
y_{n}>0 \text { and } \Delta y_{n}>0 \text { for all } n \geq N
$$


As in the proof of Theorem 1, equation (8) is then valid.

Since $g$ is non-increasing, we have from Lemma 1

$$
g\left(\Delta y_{n}\right) \geq g\left(\frac{2}{n} y_{n+1}\right) \text { for all } n \geq 2 N .
$$

Using (13) in (8) and then applying condition $\left(c_{5}\right)$ we obtain

$$
\Delta^{2} y_{n}+K_{2} q_{n} f\left(y_{n+1}\right) g\left(\frac{2}{n}\right) g\left(y_{n+1}\right) \leq 0, \quad n \geq 2 N
$$

Multiplying (14) by $(n+1)$ and then summing from $2 N$ to $n-1$ we have

$$
\sum_{s=2 N}^{n-1} \frac{(s+1) \Delta^{2} y_{s}}{f\left(y_{s+1}\right) g\left(y_{s+1}\right)}+\sum_{s=2 N}^{n-1} K_{2}(s+1) q_{s} g\left(\frac{2}{s}\right) \leq 0
$$

Using the summation by parts formula for the first term in (15), we obtain

$$
\begin{aligned}
& \frac{n \Delta y_{n}}{f\left(y_{n}\right) g\left(y_{n}\right)}-\frac{2 N \Delta y_{2 N}}{f\left(y_{2 N}\right) g\left(y_{2 N}\right)}-\sum_{s=2 N}^{n-1} \frac{\Delta y_{s}}{f\left(y_{s+1}\right) g\left(y_{s+1}\right)} \\
& +\sum_{s=2 N}^{n-1} \frac{s \Delta\left(f\left(y_{s}\right) g\left(y_{s}\right)\right)}{f\left(y_{s}\right) f\left(y_{s+1}\right) g\left(y_{s}\right) g\left(y_{s+1}\right)}+\sum_{s=2 N}^{n-1} K_{2} q_{s}(s+1) g\left(\frac{2}{s}\right) \leq 0
\end{aligned}
$$

or

$$
\frac{n \Delta y_{n}}{f\left(y_{n}\right) g\left(y_{n}\right)}-\int_{y_{2 N}}^{y_{n}} \frac{d x}{f(x) g(x)}+K_{2} \sum_{s=2 N}^{n-1}(s+1) q_{s} g\left(\frac{2}{s}\right) \leq c
$$

where $c$ is a positive constant. Letting $n \rightarrow \infty$ in (16) we obtain a contradiction. This completes the proof.

\section{Remark. In Theorem 3}

(a) if $p_{n}=0$, then it reduces to Theorem 2 of [10]

(b) if $p_{n}=0$ and $g(u) \equiv 1$ then it reduces a special case of Theorem 4.2 of Kulenovic and Budincevic [5]

(c) if $p_{n}=0, g(u) \equiv 1$ and $f(u)=u^{\alpha}, 1<\alpha<\infty$ then it reduces to Theorem 4.1 of Hooker and Patula [4].

The difference equation

$$
\Delta^{2} y_{n}+\frac{1}{n+2} \Delta y_{n}+\frac{\left(4 n^{2}+6 n+1\right)(n+1)^{2}}{n(n+2)} y_{n+1}^{3}=0, \quad n \geq 1
$$

satisfies all conditions of Theorem 3 and hence every solution of $\left(E_{2}\right)$ is oscillatory. One such solution is $y_{n}=\frac{(-1)^{n}}{n}$. 
Corollary 4. Let conditions (5) and (11) be replaced by condition $\left(c_{6}\right)$ with $\alpha \in[1, \infty)$. Then every solution of equation (1) is oscillatory.

Proof. The proof is similar to that of Theorem 3 and hence is omitted.

Theorem 5. Let conditions $\left(c_{1}\right)-\left(c_{3}\right),\left(c_{5}\right),(2)$ and (5) hold and let in addition condition $\left(c_{6}\right)$ hold for $\alpha=1$. If there exists a positive sequence $\left\{h_{n}\right\}_{n \geq N}$ such that.

$$
\sum_{n=2 N}^{\infty} h_{n}\left[q_{n} g\left(\frac{2}{n}\right)-\left(\frac{\Delta h_{n}}{2 h_{n}}\right)^{2}\right]=\infty
$$

then all solutions of equation (1) are oscillatory.

Proof. Suppose that $\left\{y_{n}\right\}$ is a non-oscillatory solution of (1). In view of Lemma 2 there is no loss in generality in assuming that there is an integer $N \geq 0$ such that

$$
y_{n}>0 \text { and } \Delta y_{n}>0 \text { for all } n \geq N
$$

From the proof of Theorem 1 , we have the inequality (8) satisfied for all $n \geq N$. Let us define $Z_{n}=h_{n} \Delta y_{n} / y_{n}$, then from (8), we get $(n \geq N)$

$$
\Delta Z_{n} \leq \frac{-h_{n} q_{n} f\left(y_{n+1}\right) g\left(\Delta y_{n}\right)}{y_{n+1}}+\frac{\Delta h_{n} \Delta y_{n+1}}{y_{n+1}}-\frac{h_{n} \Delta y_{n} \Delta y_{n+1}}{y_{n} y_{n+1}}
$$

Since $g$ is non-increasing, we have from Lemma 1 and condition $\left(c_{5}\right)$

$$
g\left(\Delta y_{n}\right) \geq K_{2} g\left(\frac{2}{n}\right) g\left(y_{n+1}\right), \text { for all } n \geq 2 N .
$$

In view of monotonicity of $y_{n}$ and $\Delta y_{n},(19)$ and $\left(c_{6}\right)$ we see that for $n \geq 2 N$

$$
\begin{aligned}
\Delta Z_{n} & \leq-\beta K_{2} h_{n} q_{n} g\left(\frac{2}{n}\right)+Z_{n+1} \frac{\Delta h_{n}}{h_{n+1}}-Z_{n+1}^{2} \frac{h_{n}}{h_{n+1}^{2}} \\
& =-\beta K_{2} h_{n} q_{n} g\left(\frac{2}{n}\right)-\frac{h_{n}}{h_{n+1}^{2}}\left[Z_{n+1}-\frac{\Delta h_{n} h_{n+1}}{2 h_{n}}\right]^{2}+\frac{\left(\Delta h_{n}\right)^{2}}{4 h_{n}} .
\end{aligned}
$$

Hence

$$
\Delta Z_{n} \leq-\beta K_{2} h_{n} q_{n} g\left(\frac{2}{n}\right)+\frac{\left(\Delta h_{n}\right)^{2}}{4 h_{n}} \quad(n \geq 2 N) .
$$

Summing the above inequality from $2 N$ to $n$, we get

$$
Z_{n+1} \leq Z_{2 N}-\beta K_{2} \sum_{s=2 N}^{n} h_{s}\left[q_{s} g\left(\frac{2}{s}\right)-\left(\frac{\Delta h_{s}}{2 h_{s}}\right)^{2}\right]
$$

Now by (17), it is easy to see that $Z_{n}$ is essentially negative which is a contradiction. 
Remark. In Theorem 5,

(a) if $p_{n}=0$, then it reduces to Theorem 4 of [10]

(b) if $p_{n} \equiv 0$ and $g(u) \equiv 1$, then it reduces a result in Szmanda [8].

Consider the difference equation

$$
\Delta^{2} y_{n}+\frac{1}{(n+2)} \Delta y_{n}+\frac{\left(4 n^{2}+6 n+1\right)}{n^{1 / 3}(2 n+1)^{2 / 3}} f\left(y_{n+1}\right) g\left(\Delta y_{n}\right)=0
$$

where $f(u)=u^{1 / 3}$ and $g(u)=(u)^{2 / 3}$, then all conditions of Theorem 5 are satisfied. Hence every solution of $\left(E_{3}\right)$ is oscillatory. One such solution is $y_{n}=\frac{(-1)^{n}}{n}$.

Finally we establish sufficient conditions for all solutions of (1) to be oscillatory when $\phi(x, y) \equiv y$ and $\left\{p_{n}\right\}$ may be negative.

Theorem 6. Let conditions $\left(c_{1}\right),\left(c_{3}\right),\left(c_{5}\right),(2)$ and (3) hold. Suppose

$$
\begin{gathered}
\int^{ \pm \infty} \frac{d u}{f(u) g(u)}<\infty \\
f(u)-f(v)=h_{1}(u, v)(u-v): h_{1}(u, v)>\sigma_{1}>0 \\
g(u)-g(v)=h_{2}(u, v)(u-v): h_{2}(u, v)>\sigma_{2}>0
\end{gathered}
$$

for $u, v \neq 0$ and there is a constant $K>0$ and a positive non-decreasing sequence $\left\{h_{n}\right\}$ such that

$$
\Delta^{2} h_{n} \leq 0 \text { and } p_{n} \geq \frac{-K}{h_{n}} \text { for all } n \geq N \geq 0
$$

If

$$
\sum^{\infty} h_{n} q_{n} g\left(\frac{2}{n}\right)=\infty
$$

then all solutions of equation (1) are oscillatory.

Proof. Let $\left\{y_{n}\right\}$ be a non-oscillatory solution of (1) such that $y_{n}>0$ and $\Delta y_{n}>0$ for $n \geq N \geq 0$. From Lemma 1, we have

$$
g\left(\Delta y_{n}\right) \geq K_{2} g\left(\frac{2}{n}\right) g\left(y_{n+1}\right) \text { for all } n \geq 2 N .
$$

Using this inequality, equation (1) becomes

$$
\Delta^{2} y_{n}+p_{n} \Delta y_{n}+K_{2} q_{n} g\left(\frac{2}{n}\right) f\left(y_{n+1}\right) g\left(y_{n+1}\right) \leq 0 \text { for } n \geq 2 N .
$$

Mulitplying (23) by $\frac{h_{n}}{f\left(y_{n+1}\right) g\left(y_{n+1}\right)}$ and summing from $2 N$ to $n-1$, we have

$$
\sum_{s=2 N}^{n-1} \frac{h_{s} \Delta^{2} y_{s}}{f\left(y_{s+1}\right) g\left(y_{s+1}\right)}+\sum_{s=2 N}^{n-1} \frac{p_{s} h_{s} \Delta y_{s}}{f\left(y_{s+1}\right) g\left(y_{s+1}\right)}+K_{2} \sum_{s=2 N}^{n-1} q_{s} h_{s} g\left(\frac{2}{s}\right) \leq 0 .
$$


Using summation by parts formula and (20) and (21), we have

$$
\begin{aligned}
& \frac{h_{n} \Delta y_{n}}{f\left(y_{n+1}\right) g\left(y_{n+1}\right)}-\frac{h_{2 N} \Delta y_{2 N}}{f\left(y_{2 N+1}\right) g\left(y_{2 N+1}\right)}-\sum_{s=2 N}^{n-1} y_{s+1} \Delta\left[\frac{h_{s}}{f\left(y_{s+1}\right) g\left(y_{s+1}\right)}\right] \\
& +\sum_{s=2 N}^{n-1} \frac{p_{s} h_{s} \Delta y_{s}}{f\left(y_{s+1}\right) g\left(y_{s+1}\right)}+K_{2} \sum_{s=2 N}^{n-1} q_{s} h_{s} g\left(\frac{2}{s}\right) \leq 0
\end{aligned}
$$

or

$$
\begin{aligned}
& \frac{h_{n} \Delta y_{n}}{f\left(y_{n+1}\right) g\left(y_{n+1}\right)}-\frac{h_{2 N} \Delta y_{2 N}}{f\left(y_{2 N+1}\right) g\left(y_{2 N+1}\right)}+\sum_{s=2 N}^{n-1} \frac{\sigma_{1} f\left(y_{s+2}\right)+\sigma_{2} g\left(y_{s+1}\right)}{f\left(y_{s+1}\right) f\left(y_{s+2}\right) g\left(y_{s+1}\right) g\left(y_{s+2}\right)} \\
& -K \int_{y_{2 N+1}}^{y_{n}} \frac{d u}{f(u) g(u)}+K_{2} \sum_{s=2 N}^{n-1} q_{s} h_{s} g\left(\frac{2}{s}\right) \leq 0
\end{aligned}
$$

or

$$
\frac{h_{n} \Delta y_{n}}{f\left(y_{n+1}\right) g\left(y_{n+1}\right)} \leq c+K \int_{y_{2 N+1}}^{y_{n}} \frac{d u}{f(u) g(u)}-K_{2} \sum_{s=2 N}^{n-1} q_{s} h_{s} g\left(\frac{2}{s}\right)
$$

where $c$ is a constant. Taking the limit as $n \rightarrow \infty$ and using (22), we arrive at the contradiction that $\Delta y_{n}<0$ for all $n \geq 2 N$. This completes the proof.

Remark. Let $h_{n}=n$, and $g(u) \equiv 1$, then Theorem 6 is a discrete analogue of Theorem 4 of Naito [6].

The equation

$$
\Delta^{2} y_{n}-\frac{1}{n} \Delta y_{n}+12(4)^{n}\left(\frac{3}{2}+\frac{1}{n}\right) y_{n+1}^{3}=0
$$

has the oscillatory solution $y_{n}=(-1)^{n} n$, since all conditions of Theorem 6 are satisfied.

\section{References}

[1] R. P. Agarwal, Difference Equations and Inequalities, Marcel Dakker, New York, 1992.

[2] J. W. Baker, "Oscillation theorems for a second order damped non-linear differential equation," SIAM J. Appl Math., 25 (1973), 37-40.

[3] S. R. Grace and B. S. Lalli, "Oscillatory behavior of nonlinear second order differential equations with deviating arguments," Bull. Inst. Math. Acad. Sinica, 14 (1986), 187-196.

[4] J. W. Hooker and W. T. Patula, "A second order non-linear difference equation: Oscillation and asymptotic behaviour," J. Math. Appl., 91 (1983), 9-24.

[5] M. R. S. Kulenovic and Budincevic, "Asymptotic Analysis of non-linear second order difference equation," Sti. Univ. Iasi, 30 (1984), 39-52.

[6] M. Naito, "Oscillation theorems for a damped non-linear differential equation," Proc. Japan, Acad., 50 (1974), 104-108.

[7] J. Popenda, "Oscillation and non-oscillation theorems for second order difference equations," $J$. Math. Anal. Suppl., 123 (1987), 34-38. 
[8] B. Szmanda, "Oscillation criteria for second order non-linear difference equations," Ann. Poln. Math., 43 (1983), 225-235.

[9] B. Szmanda, "Oscillation theorems for non-linear second order difference equations," J. Math. Anal. Appl., 79 (1981), 90-95.

[10] E. Thandapani, "Oscillation criteria for certain second order difference equations," ZAA, 11 (1992), 425-429.

Department of Mathematics, Madras University P. G. Centre, Salem-636 011, Tamil Nadu, INDIA. 